\title{
Crystal structures of dengue virus protein revealed ED3 sero-specificity
}

Manjiri Ravindra Kulkarni ${ }^{1}$, Monirul Islam ${ }^{2}$, Nobutaka Numoto ${ }^{3}$, Nobutoshi Ito $^{3}$, Yutaka Kuroda 4

${ }^{1}$ Department of Comparative Biosciences, University Of Illinois, Urbana-Champaigne, Urbana, United States, ${ }^{2}$ Department of Biochemistry and Molecular Biology, University of Chittagong, Chittagong, Bangladesh, ${ }^{3}$ Department of Structural Biology, Medical Research Institute, Tokyo Medical and Dental University, Tokyo, Japan, ${ }^{4}$ Department of Biotechnology and Life Sciences, Tokyo University of Agriculture and Technology, Tokyo, Japan

E-mail: jaimalhar.mrk@gmail.com

Dengue (DEN) fever is a re-emerging tropical disease. Although, the four serotypes (DEN1-DEN4) have 70-80\% sequence and high structural similarity, slight variations present in different serotypes are responsible for the sero-specific nature of individual serotype, which cause a life-threatening dengue hemorrhagic fever and dengue shock syndrome. Here, we aim at characterizing the sero-specificity of the immune response against DEN3 and DEN4, envelope domain 3 (ED3) through structural and biophysical analysis. ED3 contains the two major putative epitopes (E1 and E2) [1] and is a highly suitable model protein for examining the molecular determinants of a virus' sero-specificity. We designed six epitope grafted ED3 variants where the surface-exposed epitope residues of E1 and E2 from DEN3 ED3 were switched to those of DEN4 ED3 and vice versa individually and in combination by hypothesizing that sero-specificity would be transferred by switching the epitope residues. Synthetic genes encoding the ED3 sequences of DEN3 and DEN4 were cloned into a pET15b vector, overexpressed and proteins were purified. Immunizing Swiss-Albino mice produced anti-DEN3 ED3 and anti-DEN4 ED3 sera. Interaction strengths were monitored by ELISA using purified ED3s and both anti-sera. Finally, crystal structure of DEN4ED3's epitope grafted mutant where E2 residues are mutated from DEN3 ED3 (DEN4_E2DEN3 hereafter, PDB ID: 4 X42) was determined [2] by molecular replacement using DEN4 ED3 structure. Interaction patterns were further confirmed using high-resolution crystal structures of DEN3 ED3 (PDB ID: 3VTT), DEN4 ED3 (PDB ID: 4WE1) [3] and DEN4_E2DEN3 plus electrostatic surface potentials (ESPs) of other modeled grafted mutants. Mutants that were difficult to crystallize were modeled using DEN3 ED3 and DEN4 ED3 as template. We found individual protein domain ED3 gives highly sero-specific immune responses in mice. Reactivity against epitope-grafted mutants indicated that E1 in DEN4 ED3 and E2 in DEN3 ED3 play a comparatively major role in determining sero-specificity. Most importantly, crystal structures and ESPs gave an additional insight into sero-specific nature of E1 and E2. The structure of DEN4_E2DEN3 was essentially identical to that of DEN4 ED3 (RMSD: $0.33 \AA$ ) despite E2's residues being substituted from DEN3 ED3, stating that E1 plays a major role in sero-specificity of DEN4 ED3. Moreover, similar structures and ESPs of DEN4 ED3 and DEN4_E2DEN3 confirmed the similarity of interaction patterns shown by these two antigens against both anti-sera in ELISA. To sum up, detailed examination of the structures and electrostatic surfaces nevertheless provided insights into the sero-specificity of DEN ED3s that were unnoticed from sequence.

[1]Lisova. O. et al. (2007) J. Gen. Virol. 88, 2387-2397.

[2]Kulkarni. M. et al. (2015) Biochimica Biophysica Acta 1854, 1438-1443.

[3]Elahi. M. et al. (2013) Proteins 81, 1090-1095.

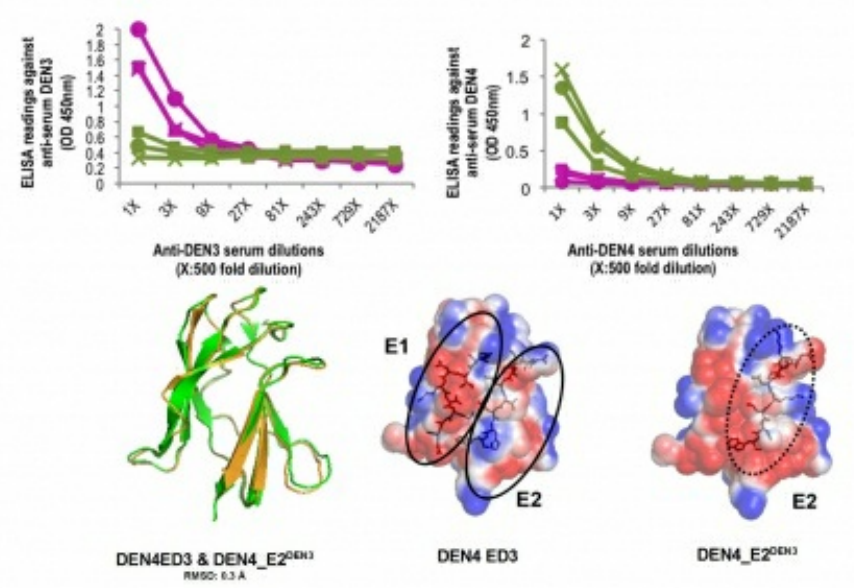

Keywords: Sero-specificity, Molecular modeling, X-ray crystallography 